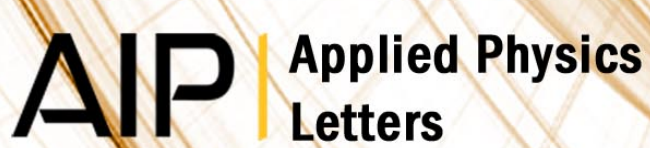

\section{Addressable subwavelength grids of confined light in a multislotted nanoresonator}

B. Cluzel, K. Foubert, L. Lalouat, J. Dellinger, D. Peyrade et al.

Citation: Appl. Phys. Lett. 98, 081101 (2011); doi: 10.1063/1.3555489

View online: http://dx.doi.org/10.1063/1.3555489

View Table of Contents: http://apl.aip.org/resource/1/APPLAB/v98/i8

Published by the American Institute of Physics.

\section{Related Articles}

Coupled plasmonic modes in organic planar microcavities

APL: Org. Electron. Photonics 5, 128 (2012)

Coupled plasmonic modes in organic planar microcavities

Appl. Phys. Lett. 100, 253301 (2012)

Parametric noise squeezing and parametric resonance of microcantilevers in air and liquid environments Rev. Sci. Instrum. 83, 065109 (2012)

Experimental demonstration of ultra-compact directional couplers based on silicon hybrid plasmonic waveguides Appl. Phys. Lett. 100, 241105 (2012)

Parametric optomechanical oscillations in two-dimensional slot-type high-Q photonic crystal cavities Appl. Phys. Lett. 100, 211908 (2012)

\section{Additional information on Appl. Phys. Lett.}

Journal Homepage: http://apl.aip.org/

Journal Information: http://apl.aip.org/about/about_the_journal

Top downloads: http://apl.aip.org/features/most_downloaded

Information for Authors: http://apl.aip.org/authors

\section{ADVERTISEMENT}

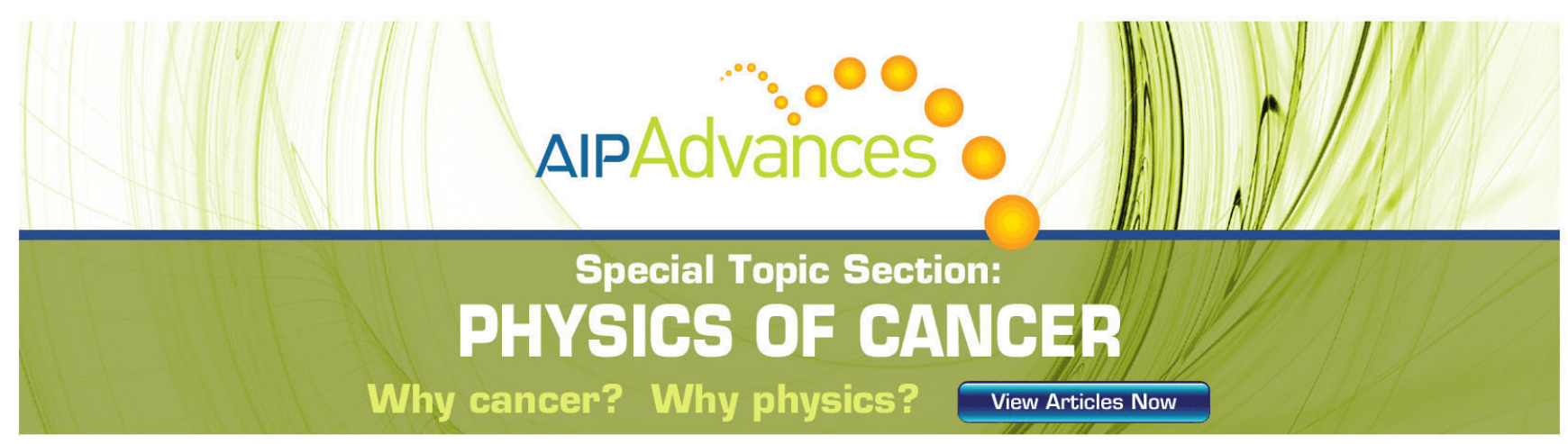




\title{
Addressable subwavelength grids of confined light in a multislotted nanoresonator
}

\author{
B. Cluzel, ${ }^{1, a)}$ K. Foubert, ${ }^{1,2}$ L. Lalouat, ${ }^{1}$ J. Dellinger, ${ }^{1}$ D. Peyrade ${ }^{3}$ E. Picard, ${ }^{2}$ E. Hadji, ${ }^{2}$ \\ and F. de Fornel ${ }^{1}$ \\ ${ }^{1}$ Groupe d'Optique de Champ Proche-LRC CEA $n^{\circ}$ DSM-08-36, Laboratoire Interdisciplinaire Carnot de \\ Bourgogne, UMR CNRS n5209-Université de Bourgogne, 9, av. A. Savary, 21078 Dijon, France \\ ${ }^{2}$ Laboratoire Silicium Nanoélectronique Photonique et Structures, INAC/SP2M/SiNaPS, CEA, 17, rue des \\ Martyrs, 38054 Grenoble, France \\ ${ }^{3}$ Laboratoire des Technologies de la Microélectronique, CNRS, 38054 Grenoble, France
}

(Received 2 September 2010; accepted 7 December 2010; published online 22 February 2011)

\begin{abstract}
In this letter, we fabricate a multislotted optical nanoresonator with several spatial field distributions which are all addressable by the wavelength. The reported structure consists in an array of evanescently coupled single mode photonic crystal nanocavities. By using a scanning near-field optical microscope, we quantify the morphology of the different optical mode volumes and show that they consist in grids of light confined at the subwavelength scale. (C) 2011 American Institute of Physics. [doi:10.1063/1.3555489]
\end{abstract}

The optical cavities miniaturized at the submicrometer scale have enabled a wide range of applications ranging from quantum information processing ${ }^{1-3}$ to biological sensing. ${ }^{4}$ Their ability to confined the light in an extremely small volume where the light matter interactions are enhanced, ${ }^{5}$ where the light is trapped ${ }^{6}$ or slowed down ${ }^{7}$ has motivated extensive researches over the past decades. In these systems, the two properties that are desired, but often incompatible, are very high quality factors (Q) and very small mode volumes (V). Among the potential optical cavities operating at this scale $^{8}$ the photonic crystal (PC) cavities ${ }^{6,9-11}$ offer a good compromise between these two features and confinement volumes at the wavelength scale are achieved thanks to their photonic band gap. Over the past decade, the advances in the field of nanotechnology fabrication facilities and in the understanding of the confinement processes inside PCs have led to cavities with Q-factors as high as several millions. Among these cavities, the one-dimensional PC nanocavities integrated on a single mode dielectric waveguide ${ }^{9,12-14}$ also combine to the previous features an extremely small mass and intense optical forces in the surrounding free space, which are of a great interest in the emerging fields of optomechanics, ${ }^{15-18}$ optical trapping, ${ }^{19}$ and optical sensing at the nanoscale. ${ }^{20,21}$

If the applications cited below require a strong field confinement inside a small cavity volume, it is also of a prime importance to consider how the light is distributed within this volume at the subwavelength scale. As a matter of fact, the light matter interactions are enhanced where the antinodes of the field are located otherwise they are inhibited. ${ }^{3,22,23}$ The optical forces are strong where the field gradient is high ${ }^{24-26}$ and a submicrometer sized object can be manipulated or detected if it overlaps a field localized at the same scale. ${ }^{27-31}$ As a consequence, an active and accurate control of these field localizations at the subwavelength scale inside the cavity volume is highly desirable to envisage the future development of configurable nanosystems. Unfortu-

\footnotetext{
a) Author to whom correspondence should be addressed. Electronic mail: benoit.cluzel@u-bourgogne.fr.
}

nately, this is basically incompatible with an ultra small cavity since it is a single mode resonator, which implies that the associated field distribution is always unique and predefined by the initial design.

In this work, we challenge this limitation by evanescently coupling several single mode PC nanocavities. This permits to fabricate a small multimodal slotted nanocavity with several spatial field distributions which are all addressable by changing the operating wavelength. In this configuration, we manage to configure the spatial distribution of the mode volumes while preserving the field confinement inside them. Using a scanning near-field optical microscope (SNOM) operating in interaction-scanning-mode, ${ }^{32}$ we provide an experimental demonstration of our claims.

The system considered here is shown in Fig. 1 and was processed on a silicon-on-insulator wafer using an electronbeam lithography followed by an inductive coupled plasma etching step. It consists in a lateral chain of eight "FabryPerot-like" cavities in which the light is loaded by evanescent coupling. Each individual cavity is defined by two PC tapered mirrors designed to improve the quality factor (Q) inside the cavity volume $(\mathrm{V}) .^{12}$ The whole is integrated on a $500 \mathrm{~nm} \times 340 \mathrm{~nm}$ silicon waveguide terminated by two antireflection tapers. In this configuration, an individual cavity exhibits a single resonance at telecommunication wavelength $(\lambda=1.5 \mu \mathrm{m})$. If such cavities potentially achieve $\mathrm{Q} / \mathrm{V}$ factors, which rival the best two-dimensional PC cavities, ${ }^{14,33}$ we note that those considered in this work are designed to operate in a moderate $\mathrm{Q}$ regime $\left(10^{3}-10^{4}\right)$ in order to achieve a robust coupling between them. The coupling between waveguides (and thus between the cavities) is achieved through a 100-nm-wide air gap, which is the best trade-off between the coupling strength and the fabrication reproducibility. To provide light injection and collection inside the structures, a rib waveguide is evanescently coupled on each side of the structure.

Then, the optical characterization has been performed in far-field by using an endfire coupling scheme. ${ }^{34}$ A transverse electrical polarized tunable laser source is coupled to the input waveguide thanks to a high numerical aperture objec- 

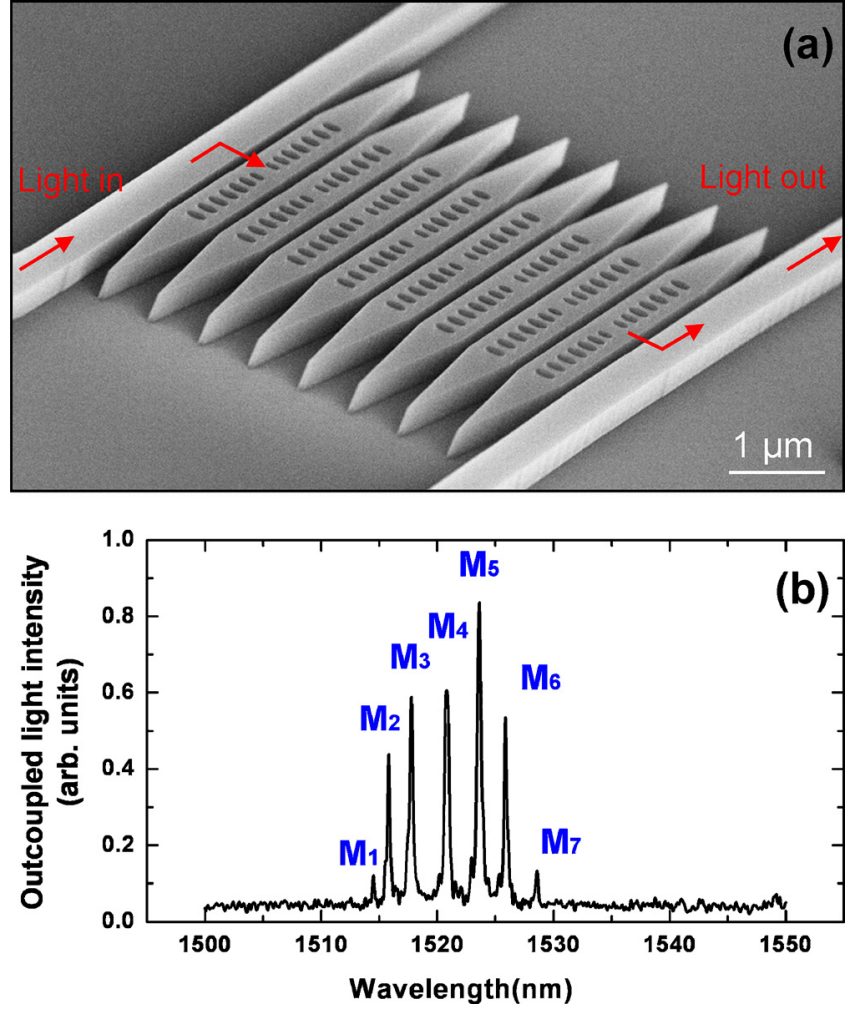

FIG. 1. (Color online) (a) Scanning electron microscope view of the multislotted resonator made of coupled nanocavities. The view includes the rib waveguides for light input and output. The arrows provide a schematic illustration of the light path through the structures. (b) Experimental spectrum of the light transmitted through the structure and outcoupled into the output waveguide.

tive and the light outcoming from the output waveguide after passing through the coupled cavity system is collected thanks to another objective. The transmission spectra of the structures are then measured by scanning the laser from 1500 to $1550 \mathrm{~nm}$ while detecting the outcoupled light intensity with a cooled photodetector. In Fig. 1(b), we show the experimental output spectrum of the system. It exhibits several well-defined transmission peaks which all correspond to a resonance of the coupled cavity system, i.e., a mode with a well defined field distribution.

To directly visualize the spatial distribution of the field confinement above the structure, we resort to the SNOM operating in the interaction scanning mode developed in our earlier works. ${ }^{32,35}$ A near-field probe consisting in a pulled optical fiber with a $20 \mathrm{~nm}$ apex is scanned at $4 \mathrm{~nm}$ above the structures thanks to a shear-force feedback while the modifications of the far-field transmittance spectrum due to the local perturbation of the resonance wavelength by the presence of the near-field probe are recorded. The resulting image is proportional to the optical near-field distribution of the considered resonant mode. ${ }^{35}$ Repeating the measurements at all the resonance wavelengths permits us to map the electromagnetic field associated to each modes. The experimental images recorded by this procedure are reported in Fig. 2. For sake of simplicity, a unique colorbar with arbitrary units is used for all the images in such a way that they can be directly compared without considering their relative intensities. A rapid glance at these images clearly shows that each resonance of the spectrum is associated to a given field distribution. One can note that some intense electromagnetic field localizations appear periodically inside the air gap between the nanocavities and also that the individual cavities can be switched on or off depending on the considered resonance. The coupling scheme between the individual cavities that leads to the specific field distribution reported here basically relies on the coupled mode theory ${ }^{36}$ and will be detailed elsewhere since this is beyond the scope of this letter.

To emphasize and quantify the subwavelength features of the reported field distributions, we plotted in Fig. 3 their lateral and longitudinal cross-sections. As illustrated in Fig. 3(a), the longitudinal field distribution (along the waveguide) is always the same whatever the considered resonant mode and corresponds to the standing wave of an individual cavity. ${ }^{32}$ The pitch of the light grid along this direction is 570 nm, i.e., $0.19 \lambda_{0}$, where $\lambda_{0}$ is the free space wavelength. Along the other direction [Fig. 3(b)], this intensity above the cavities is modulated from one mode to another one. This particular feature is visible on the different transversal crosssections as well as on the images of Fig. 2. We note here that this experimental observation is a direct evidence of a phenomenon due to the evanescent coupling between the waveguides which was predicted by Yariv and Yeh in Ref. 37. In addition, along the same direction, one can also observe that the air slots between the cavities can be enlightened or not which is associated to the constructive or destructive interferences between the field inside two adjacent cavities. ${ }^{20}$ As a consequence, the lateral field distribution of all the modes results from a combination of these two pat-
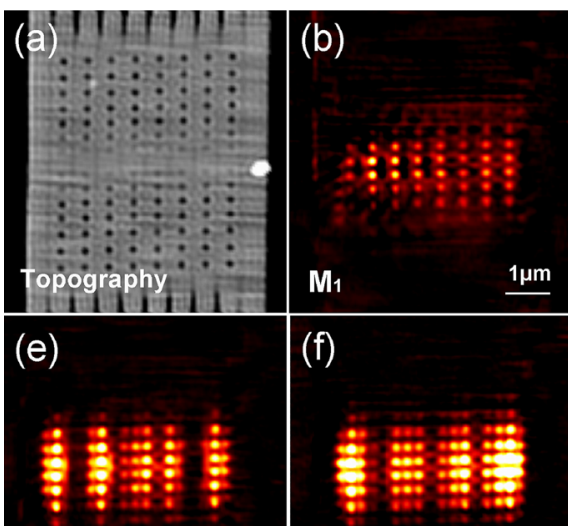

M4

$1 \mu \mathrm{m}$

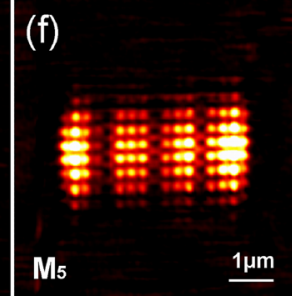

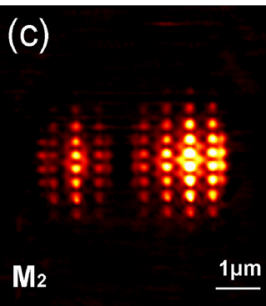

(g)

M
FIG. 2. (Color online) Experimental near-field images of the multislotted resonator. (a) Topographical image recorded using a shear-force feedback and $[(\mathrm{b})-(\mathrm{h})]$ corresponding optical near-field images of the seven resonant modes of the system recorded using the interaction scanning mode of the microscope. 
(a)

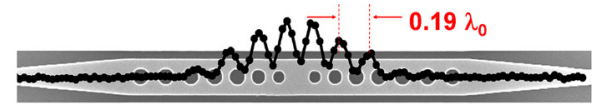

(b)
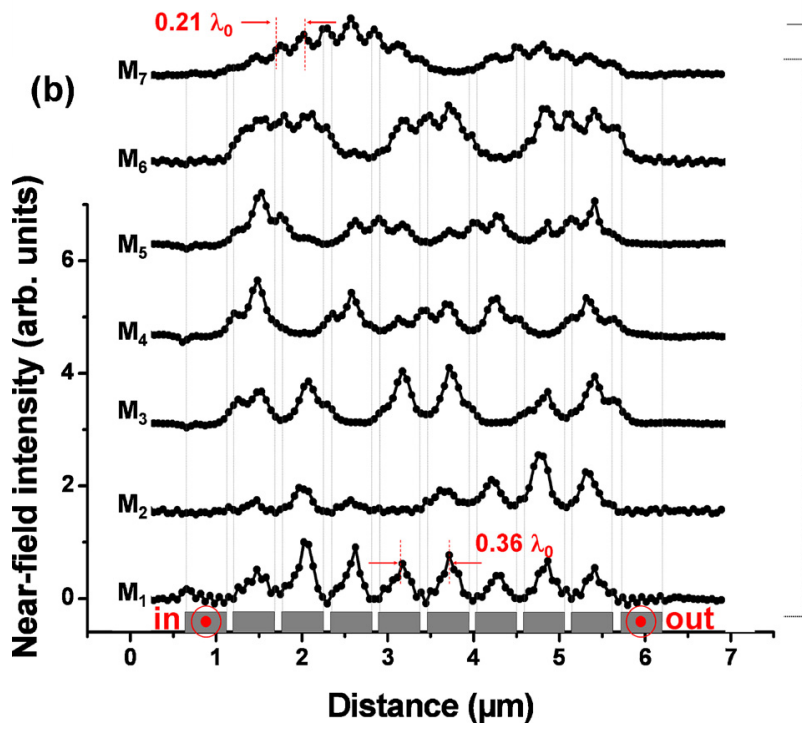

FIG. 3. (Color online) (a) Longitudinal and (b) lateral cross sections of the optical near-field images of the multislotted resonator. The seven lateral cross-sections of the seven modes are plotted since they are all different and only a single longitudinal cross-section is plotted since they are similar for all the modes. The typical pitch of the optical grids is indicated in unit of the free space wavelength to provide a direct observation of the subwavelength features of the light distribution.

terns and leads to different grids of confined light with the two characteristics pitches of $0.21 \lambda_{0}$ and $0.36 \lambda_{0}$. The combination of these two patterns, longitudinal and lateral, leads to the measured two dimensional grids of confined light which were reported in Fig. 2. In all the directions, all the grids exhibit a well defined subwavelength structure and each grid can be addressed by the operating wavelength. As a consequence, changing the operating wavelength of the coupled cavity system permits to enlighten on demand a given set of subwavelength air gaps and cavities and thus to control their related properties, the resulting optical forces for instance or the sensing channels for biological applications.

As a conclusion, we reported here the optical near-field properties of a multislotted optical resonator made of height coupled nanocavities integrated on silicon waveguides. The far-field transmission spectrum of the structure has enabled to identify its resonance wavelengths and high resolution near-field images of the field distribution within the coupled cavity system have been systematically performed. The analysis of the reported results has revealed that each resonance is associated to a well defined grid of confined light with a specific subwavelength structure. All these subwavelength grids of light are addressable by changing the wavelength which offers perspectives to achieve on a chip an active control of the spatial distribution of optical forces, traps, detection volumes, and to develop a new class of configurable optomechanical nanosystems.
${ }^{1}$ T. Yoshie, A. Scherer, J. Hendrickson, G. Khitrova, H. M. Gibbs, G. Rupper, C. Ell, O. B. Shchekin, and D. G. Deppe, Nature (London) 432, 200 (2004).

${ }^{2}$ J. P. Reithmaier, G. Sek, A. Löffler, C. Hofmann, S. Kuhn, S. Reitzenstein, L. V. Keldysh, V. D. Kulakovskii, T. L. Reinecke, and A. Forchel, Nature (London) 432, 197 (2004).

${ }^{3}$ K. Hennessy, A. Badolato, M. Winger, D. Gerace, M. Atature, S. Gulde, S. Falt, E. L. Hu, and A. Imamoglu, Nature (London) 445, 896 (2007).

${ }^{4}$ A. H. J. Yang, S. D. Moore, B. S. Schmidt, M. Klug, M. Lipson, and D. Erickson, Nature (London) 457, 71 (2009).

${ }^{5}$ M. SoljaČiĆ and J. D. Joannopoulos, Nature Mater. 3, 211 (2004).

${ }^{6}$ B. S. Song, S. Noda, T. Asano, and Y. Akahane, Nature Mater. 4, 207 (2005).

${ }^{7}$ M. Notomi, E. Kuramochi, and T. Tanabe, Nat. Photonics 2, 741 (2008).

${ }^{8}$ See for a review of the cavity geometries, K. J. Vahala, Nature (London) 424, 839 (2003).

${ }^{9}$ J. S. Foresi, P. R. Villeneuve, J. Ferrera, E. R. Thoen, G. Steinmeyer, S. Fan, J. D. Joannopoulos, L. C. Kimerling, H. I. Smith, and E. P. Ippen, Nature (London) 390, 143 (1997).

${ }^{10}$ Y. Akahane, T. Asano, B.-S. Song, and S. Noda, Nature (London) 425 944 (2003).

${ }^{11}$ T. Tanabe, M. Notomi, E. Kuramochi, A. Shinya, and H. Taniyama, Nat. Photonics 1, 49 (2007).

${ }^{12}$ P. Velha, J. C. Rodier, P. Lalanne, J. P. Hugonin, D. Peyrade, E. Picard, T. Charvolin, and E. Hadji, Appl. Phys. Lett. 89, 171121 (2006).

${ }^{13}$ P. Deotare, M. W. McCutcheon, I. W. Frank, M. Khan, and M. Loncar, Appl. Phys. Lett. 94, 121106 (2009).

${ }^{14}$ A. R. Md Zain, N. P. Johnson, M. Sorel, and R. De La Rue, Opt. Express 16, 12084 (2008).

${ }^{15}$ M. Eichenfield, R. Camacho, J. Chan, K. J. Vahala, and O. Painter, Nature (London) 459, 550 (2009).

${ }^{16}$ M. Eichenfield, R. Camacho, J. Chan, K. J. Vahala, and O. Painter, Nature (London) 462, 78 (2009).

${ }^{17}$ T. J. Kippenberg and K. J. Vahala, Opt. Express 15, 17172 (2007).

${ }^{18} \mathrm{M}$. Li, W. H. P. Pernice, C. Xiong, T. Baehr-Jones, M. Hochberg, and H. X. Tang, Nature (London) 456, 480 (2008).

${ }^{19}$ S. Mandal, X. Serey, and D. Erickson, Nano Lett. 10, 99 (2010).

${ }^{20}$ K. Foubert, L. Laouat, B. Cluzel, E. Picard, D. Peyrade, F. de Fornel, and E. Hadji, Appl. Phys. Lett. 94, 251111 (2009).

${ }^{21}$ A. Di Falco, L. O'Faolain, and T. F. Krauss, Appl. Phys. Lett. 94, 063503 (2009).

${ }^{22}$ E. Yablonovitch, Phys. Rev. Lett. 58, 2059 (1987).

${ }^{23}$ E. M. Purcell, Phys. Rev. 69, 681 (1956).

${ }^{24}$ A. Ashkin, Phys. Rev. Lett. 24, 156 (1970).

${ }^{25}$ M. L. Povinelli, M. Loncar, M. Ibanescu, E. J. Smythe, S. G. Johnson, F. Capasso, and J. D. Joannopoulos, Opt. Lett. 30, 3042 (2005).

${ }^{26}$ P. T. Rakich, M. A. Popovic, M. Soljacic, and E. P. Ippen, Nat. Photonics 1, 658 (2007)

${ }^{27}$ R. Gómes-Medina, P. San José, A. Garcia-Martin, M. Lester, M. NietoVesperinas, and J. J. Sàenz, Phys. Rev. Lett. 86, 4275 (2001).

${ }^{28}$ L. C. Maier and J. C. Slater, J. Appl. Phys. 23, 68 (1952).

${ }^{29}$ A. Gokirmak, D.-H. Wu, J. S. A. Bridgewater, and S. M. Anlage, Rev. Sci. Instrum. 69, 3410 (1998).

${ }^{30}$ A. Lewis, H. Taha, A. Strinkovski, A. Manevitch, A. Khatchatouriants, R Dekhter, and E. Ammann, Nat. Biotechnol. 21, 1378 (2003).

${ }^{31}$ S. Lal, S. Link, and N. J. Halas, Nat. Photonics 1, 641 (2007).

${ }^{32}$ L. Lalouat, B. Cluzel, F. de Fornel, P. Vehla, P. Lalanne, D. Peyrade, E. Picard, T. Charvolin, and E. Hadji, Appl. Phys. Lett. 92, 111111 (2008).

${ }^{33}$ P. Velha, E. Picard, T. Charvolin, E. Hadji, J. C. Rodier, P. Lalanne, and D. Peyrade, Opt. Exp. 15, 16090 (2007).

${ }^{34}$ M. Zelsmann, E. Picard, T. Charvolin, E. Hadji, B. Dal Zotto, M. E. Nier, C. Seassal, P. Rojo-Romeo, and X. Letartre, Appl. Phys. Lett. 81, 2340 (2002).

${ }^{35}$ L. Lalouat, B. Cluzel, P. Velha, E. Picard, D. Peyrade, J. P. Hugonin, P. Lalanne, E. Hadji, and F. de Fornel, Phys. Rev. B 76, 041102 (2007).

${ }^{36}$ A. Yariv, IEEE J. Quantum Electron. 9, 919 (1973).

${ }^{37}$ A. Yariv and P. Yeh, Optical Waves in Crystals: Propagation and Control of Laser Radiation (Wiley, New York, 1983). 